\title{
Home Treatment - aufsuchende Behandlung im Hamburger Modell der Integrierten Versorgung
}

\author{
Home Treatment - A Treatment Model of Integrated Care in Hamburg
}

Autoren

Institut
Daniel Schöttle, Friederike Ruppelt, Anne Karow, Martin Lambert

Klinik für Psychiatrie und Psychotherapie, Universitätsklinikum Hamburg-Eppendorf
Schlüsselwörter

- Assertive Community Treatment

- Psychose

- schwere psychische Erkrankung

Keywords

- assertive community treatment

- psychosis

- severe mental illness

eingereicht 4. April 2014

akzeptiert 9. September 2014

Bibliografie

DOI http://dx.doi.org/

10.1055/s-0034-1390424

Online-Publikation: 8.12.2014

Psychother Psych Med 2015;

65: 140-145

(c) Georg Thieme Verlag KG

Stuttgart · New York

ISSN 0937-2032

Korrespondenzadresse

Dr. med. Daniel Schöttle

Zentrum für Psychosoziale

Medizin, Klinik für Psychiatrie

und Psychotherapie

Universitätsklinikum

Hamburg-Eppendorf

Martinistraße 52

20246 Hamburg

d.schoettle@uke.de

\section{Zusammenfassung}

Aufsuchende Behandlungsformen, wie das „Crisis Resolution and Home Treatment (CRHT)“ oder „Assertive Community Treatment“ (ACT), haben sich als effektive und die Behandlungsqualität verbessernde Modelle zur Behandlung von Menschen mit schweren psychischen Erkrankungen erwiesen. In Deutschland sind diese noch wenig implementiert, als Bestandteil einer sektorenübergreifenden Integrierten Versorgung jedoch vereinzelt vorhanden. Anhand des Hamburger Modells wird dargestellt, wie die Umsetzung einer solchen Behandlung durch ein ACT-Team als Teil der Integrierten Versorgung (IV) aussehen kann und anhand der Ergebnisse des 3-Jahres-Verlaufs diskutiert. Das Hamburger Modell bezeichnet ein IV-Modell für schwer erkrankte Psychose-Patienten. Das Modell wird seit 2007 als Managed Care „Capitation-Modell“ über den $\$ 140$ SGB V als Jahrespauschale von verschiedenen Krankenkassen finanziert und kontinuierlich bezüglich der Effektivität und Effizienz untersucht. In Vorstudien zu diesem Modell zeigte sich bereits bei Kostenneutralität im Vergleich zur Standardbehandlung eine niedrige Gesamtbehandlungsabbruchrate sowie signifikante Verbesserungen hinsichtlich Psychopathologie, Funktionsstatus, Lebensqualität, Therapieadhärenz und Behandlungszufriedenheit. Auch die Rate von Zwangseinweisungen verringerte sich signifikant auf $10 \%$ im Vergleich zu den Vorjahren. 2011 wurde das Modell im Rahmen einer vom Bundesministerium für Bildung und Forschung (BMBF) geförderten Studie (Integrated Care in Early Psychosis, ICEP-Studie) auf die Indikation „ersterkrankte Jugendliche und junge Erwachsene im Alter von 12 bis 29 Jahren" spezifiziert. Diesbezüglich wurde ein fachübergreifendes Team implementiert. Dieses Projekt wird seit 2012 von den beteiligten Krankenkassen gemäß einer Erweiterung des $§ 140$ SGB V finanziert.

\section{Abstract \\ $\nabla$}

Treatment models like "Crisis Resolution and Hometreatment (CRHT)" or "Assertive Community Treatment" (ACT), were found to be effective, enhancing the qualitative level of treatment for patients with severe mental disorders. In Germany, these are implemented only sporadically until today, often as part of a cross-sectoral Integrated Care (IC) treatment system. We will present the implementation of an "Assertive Community Treatment" embedded into an IC-treatment model in Hamburg and discuss the 3-year-outcomes. The IC-treatment model has been designed for severe mentally ill patients with psychotic disorders. Since May 2007 the model is financed by different health insurances as a managed-care "capitation-model" and its effectiveness gets continuously evaluated. The model proved to be effective in earlier studies were compared with standard care low rates of service disengagement were found as well as significantly improved psychopathology, psychosocial functioning, quality of life, satisfaction with care and adherence, while being cost effective. The rates of involuntary admissions declined to $10 \%$ in comparison to the years before. In 2011 the model was specified to the indication "first-episode adolescents and young adults in the age of 12-29" in a government-funded study "Integrated Care in Early Psychosis, ICEP Study". In this study an interdisciplinary team of child, adolescent and adult psychiatrists was implemented and since 2012 it is financed by the involved health insurances throughout an expansion of the $\S 140$ SGB V agreement. 


\section{Einleitung}

\section{$\nabla$}

Die Versorgung von Menschen mit schweren psychischen Erkrankungen (engl. Severe Mental Illness, SMI) stellt die Behandler aufgrund der psychosozialen Komplexität der Erkrankungen vor vielfältige Herausforderungen [1]. Oft beginnen diese in einer besonders vulnerablen Phase des Lebens zwischen dem 12. und 25. Lebensjahr; 20-30\% der Psychose-Betroffenen erkrankten vor ihrem 18. Lebensjahr [2-4].

Bei psychotischen Erkrankungen kommt es häufig zu einer Behandlungsverzögerung von ca. 5-6 Jahren $[2,3,5]$, welche weitreichende negative Auswirkungen auf Verlauf und Prognose haben kann [2,3,6-8]). Häufig bestehen zudem schon bei Erstkontakt komplexe soziale Probleme [2,3,9,10].

Der Verlauf ist oft von rezidivierenden Episoden bzw. einer unzureichenden interepisodischen Remission und einhergehenden sozialen Einbußen gekennzeichnet; ca. 90\% der Schizophrenie-Betroffenen erfüllen die Kriterien für eine SMI [11]. Neben der Behandlungsverzögerung sind wesentliche Ursachen dieses Erkrankungsverlaufs ein oft unzureichendes Behandlungsangebot bzw. eine unzureichende Therapieinanspruchnahme [12-15].

\section{Modelle aufsuchender Behandlung}

Um den oben genannten Anforderungen an die Bedürfnisse von Menschen mit SMI gerecht zu werden, wurden verschiedene Modelle aufsuchender Behandlungen entwickelt. Hierzu gehören:

- (Ressource Group) Assertive Community Treatment (ACT) $[16,17]$,

- Crisis Intervention and Home Treatment (CRHT) [18, 19],

- Intensives Case Management (ICM) [20] und

- Community Mental Health Teams (CMHT) [21].

Diese Behandlungsmodelle unterscheiden sich in einer Reihe von Bereichen, v.a. Teamansatz, Intensität der Behandlung, Einbindung von Betroffenen und Angehörigen, Behandlungszeitraum und im primär aufsuchenden oder nicht aufsuchenden Charakter [22]. Insgesamt sprechen die Studien jedoch für einen deutlichen Vorteil von aufsuchenden Behandlungsmodellen [16-22]. Zudem zeigte sich in den wenigen vorliegenden Studien meist zumindest eine Kostenneutralität, bzw. Kostenreduktion im Vergleich zur Standardbehandlung bei jedoch signifikant besseren Gesundheitseffekten [22].

Die positive Studienevidenz bildet sich auch in den S3-Leitlininen „Psychosoziale Therapien bei schweren psychischen Erkrankungen“ [1] ab. In diesen wird eine „kontinuierliche, gemeindenahe, aufsuchende, teambasierte und multiprofessionelle Behandlung zur Versorgung von Menschen mit schwerer psychischer Erkrankung“ empfohlen.

\section{Das „Hamburger Modell“}

\section{Überblick}

Das „Hamburger Modell“ bezeichnet ein Integriertes Versorgungsmodell (IV-Modell) für schwer erkrankte Psychose-Patienten. Das Modell wurde 2005 evidenzbasiert entwickelt [2,9], 2006 implementiert und untersucht (ACCESS-I-Studie; [2,3]). Seit 2007 wird das Modell als „Capitation-Modell“ über den $\S 140$ SGB V als Jahrespauschale von verschiedenen Krankenkassen finanziert $[9,10]$, wobei kontinuierlich eine Evaluation von
Effektivität und Effizienz (ACCESS-II-Studie; $[10,23]$ ) stattfindet. 2011 wurde es im Rahmen einer BMBF-Studie auf die Indikation „ersterkrankte Jugendliche und junge Erwachsene im Alter von 12 bis 29 Jahren“ spezifiziert und wird ebenfalls evaluiert. Seit 2012 wurde von den beteiligten Krankenkassen der Erweiterung des $§ 140$ SGB V auf diese Indikation zugestimmt.

\section{Indikationen zur Teilnahme}

Die Indikationen zur Teilnahme sind in $\bullet$ Tab. 1 dargestellt.

\section{Inhalte der Behandlungsmodelle}

Wie oben beschrieben existiert das Hamburger Modell derzeit in 2 Spezifizierungen: (1) für mehrfacherkrankte Patienten $\geq 18$ Jahren und

(2) für ersterkrankte Jugendliche und junge Erwachsene im Alter von 12 bis 29 Jahren.

Die strukturelle und inhaltliche Ausgestaltung unterscheidet sich zwischen den Modellen ( $\bullet$ Tab. 2).

Kernbestandteil beider Modelle ist das sog. Assertive Community Treatment (ACT). Dies umfasst eine „aufsuchende, intensive und langfristige Behandlung für Menschen mit schweren und persistierenden psychischen Erkrankungen“" [16,24]. Wichtige Kernmerkmale der ACT-Behandlung sind u.a. multiprofessionelles Team, niedrige Behandler-Patienten-Ratio, „No Drop Out Policy“ (Abbruch der Behandlung soll vermieden werden), 24 Stunden tägliche Erreichbarkeit und Behandlungskontinuität.

\section{3-Jahres Ergebnisse ACCESS-II-Studie}

\section{Methodik}

Im Rahmen der ACCESS-II-Studie werden alle Patienten bei Aufnahme und dann nach 6 Wochen, 3, 6 und 12 Monaten und dann fortlaufend alle 6 Monate u.a. bezüglich Therapieadhärenz, Psychopathologie [28], Krankheitsschwere [25,26], Funktionsniveau [27], Wohn- und Arbeitssituation [29], Lebensqualität [30] und zur Zufriedenheit mit der Behandlung [31] untersucht.

\section{Ergebnisse}

Seit dem 1. Mai 2007 wurden 240 erst- und mehrfacherkrankte Patienten in das Hamburger Modell aufgenommen und für diese Analyse diejenigen mit Schizophrenie-Spektrumsstörungen (SSD) oder Bipolaren Störungen (BD) ausgewählt, die für mind. 3 Jahre in der IV behandelt wurden.

115 Patienten mit SSD oder BD (44,3\% männlich; mittleres Alter: 41,8 Jahre, Standardabweichung 12,9) wurden für die Analyse ausgewählt (Aufnahmedaten siehe $\bullet$ Tab. 3). Beide Patientengruppen waren zu Beginn schwer erkrankt, hatten eine niedrige Lebensqualität und berichteten von einer geringen Zufriedenheit mit der vorherigen Behandlung. Bis auf eine längere Dauer der unbehandelten Psychose (DUP), weniger Suizidversuche in der Vorgeschichte und eine geringere Krankheitseinsicht bei der SSD-Gruppe gab es keine Unterschiede zwischen den Patientengruppen.

\section{Abbruch der Gesamtbehandlung}

Lediglich 4 der 115 Patienten (3,5\%) brachen die Gesamtbehandlung gegen die Empfehlung der Behandler ab ( = nicht-praktische Gründe). Durchschnittlich geschah dies im Mittel nach 36,9 Wochen (Range 16,0-88,1). Weitere 21 Patienten (18,3\%) wurden aus der IV aus praktischen Gründen ausgeschlossen (z.B. neuer Wohnort). Durchschnittlich geschah dies im Mittel nach 104,4 Wochen (Range 5,9-154,9). 


\begin{tabular}{|c|c|}
\hline Indikation & Details \\
\hline $\begin{array}{l}\text { versichert bei } \\
\text { folgenden } \\
\text { Krankenkassen }\end{array}$ & $\begin{array}{l}\text { - DAK Gesundheit } \\
\text { - HEK } \\
\text { - IKK Classic } \\
\text { - AOK Rheinland/Hamburg }\end{array}$ \\
\hline $\begin{array}{l}\text { diagnostische } \\
\text { Indikation nach ICD-10 }\end{array}$ & $\begin{array}{l}\text { - F1-Störungen: } \\
\text { - substanzinduzierte psychotische Störung (F1x.5) } \\
\text { - F2-Störungen: } \\
\text { - Schizophrenie (F20) } \\
\text { - anhaltende wahnhafte Störung (F22) } \\
\text { - akute vorübergehende psychotische Störung (F23) } \\
\text { - induzierte wahnhafte Störung (F24) } \\
\text { - schizoaffektive Störung (F25) } \\
\text { - sonstige nichtorganische psychotische Störung (F28) } \\
\text { - nicht näher bezeichnende nichtorganische Psychose (F29) } \\
\text { - F3-Störungen: } \\
\text { - manische Episode mit psychotischen Symptomen (F30) } \\
\text { - bipolar affektive Störung (F31) } \\
\text { - schwere depressive Episode mit psychotischen Symptomen (F32.3) } \\
\text { - rezidivierende depressive Störung, gegenwärtig schwere depressive Episode } \\
\quad \text { mit psychotischen Symptomen (F33.3) }\end{array}$ \\
\hline Phase der Erkrankung & $\begin{array}{l}\text { - Mehrfacherkrankt } \\
\text { - ersterkrankt im Alter von } 12 \text { bis } 29 \text { Jahren }\end{array}$ \\
\hline $\begin{array}{l}\text { Indikation zur } \\
\text { stationären Behandlung }\end{array}$ & $\begin{array}{l}\text { - vorliegende Einweisung zur stationären Aufnahme von einem niedergelassenen } \\
\text { Psychiater oder bei Notfallaufnahme von einem Krankenhausarzt }\end{array}$ \\
\hline $\begin{array}{l}\text { Erfüllung des allgemeinen } \\
\text { Schweregradkriteriums }\end{array}$ & $\begin{array}{l}\text { - Erfüllung des allgemeinen Schweregrad-Kriteriums im BPRS: } \\
\text { - Mind. } 40 \text { Punkte in der Brief Psychiatric Rating Scale (BPRS) }\end{array}$ \\
\hline $\begin{array}{l}\text { Erfüllung mind. } \\
\text { eines der spezifischen } \\
\text { Schweregradkriterien }\end{array}$ & $\begin{array}{l}\text { - Erfüllung mind. eines der spezifischen Schweregradkriterien im BPRS: } \\
\text { - Halluzinationen (Item 10) } \geq 6 \text { Punkte } \\
\text { - Wahn (Item 11) } \geq 6 \text { Punkte } \\
\text { - Desorganisation (Item 15) } \geq 6 \text { Punkte } \\
\text { - depressiv-suizidales Syndrom } \geq 10 \text { Punkte } \\
\text { - suizidales Syndrom } \geq 6 \text { Punkte } \\
\text { - manisches Syndrom } \geq 15 \text { Punkte } \\
\text { - Verhaltensstörungs-Syndrom im Rahmen einer Psychose } \geq 15 \text { Punkte } \\
\text { - Syndrom vorherrschender Negativsymptomatik } \geq 15 \text { Punkte }\end{array}$ \\
\hline
\end{tabular}

Tab. 1 Indikationen zur Teilnahme am Hamburger Modell (mit Erweiterung auf ersterkrankte Jugendliche und junge Erwachsene).

Tab. 2 Strukturelle und klinische Ausgestaltung des Hamburger Modells für mehrfacherkrankte und ersterkrankte Jugendliche und junge Erwachsene.

\begin{tabular}{|c|c|c|}
\hline Merkmale & $\begin{array}{l}\text { Hamburger Modell für } \\
\text { mehrfacherkrankte Patienten }\end{array}$ & $\begin{array}{l}\text { Hamburger Modell für ersterkrankte } \\
\text { Jugendliche und junge Erwachsene }\end{array}$ \\
\hline $\begin{array}{l}\text { sektorale } \\
\text { Zuständigkeit }\end{array}$ & - Sektor der Erwachsenenpsychiatrie des UKE & $\begin{array}{l}\text { - Sektoren der Erwachsenen- und Kinder- und Jugendpsychiatrie des } \\
\text { UKE }\end{array}$ \\
\hline $\begin{array}{l}\text { übergeordnete } \\
\text { Aufgaben }\end{array}$ & - sektorenübergreifende „Open-End“-Behandlung & $\begin{array}{l}\text { - ambulante und mobile Früherkennung und sektorenübergreifende } \\
\text { „Open-End“-Behandlung }\end{array}$ \\
\hline $\begin{array}{l}\text { beteiligte } \\
\text { Institutionen } \\
\text { im UKE }\end{array}$ & - Erwachsenenpsychiatrie (EP) & $\begin{array}{l}\text { - Kinder- und Jugendpsychiatrie (KJP) und Erwachsenenpsychiatrie } \\
\text { (EP) }\end{array}$ \\
\hline $\begin{array}{l}\text { beteiligte } \\
\text { stationäre }\end{array}$ & $\begin{array}{l}\text { - Psychosen Spezialstation (EP) } \\
\text { - Akutstation (EP) }\end{array}$ & $\begin{array}{l}\text { - Psychosen Spezialstation (EP) } \\
\text { - Akutstationen (EP \& KJP) } \\
\text { - Adoleszenten Spezialstation (EP \& KJP) }\end{array}$ \\
\hline $\begin{array}{l}\text { beteiligte } \\
\text { tagesklinische } \\
\text { Institutionen }\end{array}$ & $\begin{array}{l}\text { - Tagesklinik der Psychosen Spezialstation (EP) } \\
\text { - Psychosen Spezialtagesklinik für Jugendliche } \\
\text { und junge Erwachsene (EP \& KJP) } \\
\text { - Tagesklinik der Arbeitstherapie }\end{array}$ & $\begin{array}{l}\text { - Tagesklinik der Psychosen Spezialstation (EP) } \\
\text { - Psychosen Spezialtagesklinik für Jugendliche und junge Erwachsene } \\
\text { (EP \& KJP) }\end{array}$ \\
\hline $\begin{array}{l}\text { beteiligte } \\
\text { ambulante } \\
\text { Institutionen }\end{array}$ & $\begin{array}{l}\text { - Psychosen Spezialambulanz (EP) } \\
\text { - Assertive Community Treatment Team (EP) }\end{array}$ & $\begin{array}{l}\text { - Psychosen Spezialambulanz (EP) } \\
\text { - Psychosen Ersterkennungs- und Behandlungsambulanz (EP \& KJP) } \\
\text { - Assertive Community Treatment Team (EP \& KJP) }\end{array}$ \\
\hline $\begin{array}{l}\text { Mitarbeiter } \\
\text { ACT Team }\end{array}$ & $\begin{array}{l}\text { - Mitarbeiter der EP (Ärzte, Psychologen, } \\
\text { Pflegepersonal, Sozialpädagogen) }\end{array}$ & - Mitarbeiter der EP und KJP (Ärzte, Psychologen, Sozialpädagogen) \\
\hline Netzwerkpartner & - Niedergelassene Psychiater (EP) & $\begin{array}{l}\text { - Niedergelassene Psychiater (EP \& KJP) } \\
\text { - Jugendhilfe } \\
\text { - schulpsychologischer Dienst } \\
\text { - SGB-12-Institutionen }\end{array}$ \\
\hline
\end{tabular}

$E P=$ Erwachsenenpsychiatrie; KJP=Kinder- und Jugendpsychiatrie; $E P \&$ KJP = Gemeinsame Institution der Erwachsenen- und Kinder- und Jugendpsychiatrie des UKE 
Tab. 3 Charakteristika der Patienten der ACCESS-II-Studie.

\begin{tabular}{|c|c|c|c|c|}
\hline Demografische Daten & alle Patienten $(n=115)$ & $\operatorname{SSD}(n=92)$ & $B D(n=23)$ & p-Value \\
\hline Alter, Mean (SD) & $41,8(12,9)$ & $41,4(12,8)$ & $43,6(13,2)$ & 0,47 \\
\hline Geschlecht, männlich, n (\%) & $51(44,3)$ & $41(44,6)$ & $10(43,5)$ & 0,23 \\
\hline Partnerschaft, Single, $\mathrm{n}(\%)$ & $100(87,0)$ & $83(90,2)$ & $17(73,9)$ & 0,04 \\
\hline schulische Ausbildung & & & & 0,48 \\
\hline 9 Jahre, n (\%) & $18(16,4)$ & $14(15,9)$ & $4(18,2)$ & \\
\hline 10 Jahre, n (\%) & $37(33,6)$ & $32(36,4)$ & $5(22,7)$ & \\
\hline 13 Jahre, n (\%) & $55(50,0)$ & $42(47,7)$ & $13(59,1)$ & \\
\hline abgeschlossene Ausbildung, $\mathrm{n}(\%)$ & $73(63,5)$ & $58(63,0)$ & $15(65,2)$ & 0,65 \\
\hline Beschäftigt, n (\%) & $22(18,1)$ & $18(19,6)$ & $3(13,0)$ & 0,47 \\
\hline unabhängiges Leben, n (\%) & $102(88,7)$ & $80(87)$ & $22(95,7)$ & 0,24 \\
\hline \multicolumn{5}{|l|}{ Krankheitsdetails } \\
\hline Ersterkrankung, n (\%) & $15(13,0)$ & $14(15,2)$ & $1(4,3)$ & 0,17 \\
\hline komorbide psychische Störung zu Beginn, n (\%) & $87(75,7)$ & $71(77,2)$ & $16(69,6)$ & 0,45 \\
\hline Suchterkrankung, n (\%) & $62(53,9)$ & $51(55,4)$ & $11(47,8)$ & 0,51 \\
\hline andere komorbide Störung, $\mathrm{n}(\%)$ & $68(59,1)$ & $55(59,8)$ & $13(56,5)$ & 0,77 \\
\hline \multicolumn{5}{|l|}{ Familienanamnese $^{a}$} \\
\hline psychische Störung, $\mathrm{n}$ (\%) & $54(47,0)$ & $42(45,7)$ & $12(52,2)$ & 0,78 \\
\hline psychotische Störung, n (\%) & $31(27,0)$ & $23(25,0)$ & $8(34,8)$ & 0,45 \\
\hline \multicolumn{5}{|l|}{ vorangegangene stationäre Behandlung } \\
\hline stationäre Behandlung, Lebenszeit, n (\%) & $97(84,3)$ & $76(82,6)$ & $21(91,3)$ & 0,21 \\
\hline Zwangseinweisung, Lebenszeit, $\mathrm{n}$ (\%) & $50(43,5)$ & $39(42,4)$ & $11(47,8)$ & 0,52 \\
\hline Zwangseinweisung, 2 Jahre vor IV, n (\%) & $40(34,8)$ & $30(32,6)$ & $10(43,5)$ & 0,26 \\
\hline psychotherapeutische Behandlung ${ }^{\mathrm{b}}, \mathrm{n}(\%)$ & $15(13)$ & $10(10,9)$ & $5(21,7)$ & 0,17 \\
\hline Krankheitseinsicht, $n(\%)$ & $72(61,0)$ & $51(55,4)$ & $18(81,8)$ & 0,04 \\
\hline Suizidversuche in der Vergangenheit, $\mathrm{n}$ (\%) & $47(40,9)$ & $33(35,9)$ & $14(60,9)$ & 0,03 \\
\hline \multicolumn{5}{|l|}{ traumatische Ereignis } \\
\hline jegliches traumatisches Ereignis, n (\%) & $73(63,5)$ & $55(59,8)$ & $18(78,3)$ & 0,14 \\
\hline traumatisches Ereignis vor 18. Lebensjahr, $\mathrm{n}$ (\%) & $58(50,4)$ & $47(51,1)$ & $11(47,8)$ & 0,93 \\
\hline \multicolumn{5}{|l|}{ Dauer der unbehandelten Erkrankung } \\
\hline DUI, median in Wochen (quartile) & $156,4(52,3-275,0)$ & $156,6(56,5-264,2)$ & $104,4(44,6-373,7)$ & 0,67 \\
\hline DUP, median in Wochen (quartile) & $21,6(5,9-52,1)$ & $21,9(8,4-52,1)$ & $8,4(0,0-21,9)$ & 0,01 \\
\hline volle Adhärenz bei letzter Medikation, $\mathrm{n}(\%)$ & $29(25,2)$ & $25(27,2)$ & $4(17,4)$ & 0,34 \\
\hline \multicolumn{5}{|l|}{ Skalenaufnahmewerte } \\
\hline BPRS gesamt, Mean (SD) & $81,8(20,5)$ & $81,3(19,7)$ & $84,0(23,8)$ & 0,57 \\
\hline CGI-S-score, Mean (SD) & $5,9(0,9)$ & $5,9(0,9)$ & $5,8(1,1)$ & 0,70 \\
\hline GAF-score, Mean (SD) & $37,0(12,2)$ & $36,7(12,2)$ & $38,0(12,8)$ & 0,67 \\
\hline Q-LES-Q-18-Score, Mean (SD) & $2,2(0,6)$ & $2,2(0,6)$ & $2,3(0,8)$ & 0,70 \\
\hline CSQ-8 P-Score, Mean (SD) & $1,9(0,4)$ & $1,9(0,4)$ & $2,0(0,4)$ & 0,28 \\
\hline
\end{tabular}

\section{Klinischer Verlauf, Behandlungszufriedenheit,} medikamentöse Adhärenz

Im Verlauf der 3-jährigen Behandlung kam es zu signifikanten Verbesserungen in den Bereichen

- Psychopathologie („Brief Psychiatric Rating Scale“ (BPRS); Aufnahme: 81,8 (20,5); $\mathrm{F}=8,7, \mathrm{p}<0,001)$,

- Krankheitsschwere („Clinical Global Impression - Severity Scale“ (CGI-S): Aufnahme: 5,9 (0,9); Endpunkt: 4,1 (1,1) Mean (SD); $F=10,8, p<0,001$;

- CGI-BP: Aufnahme: 5,8 (1,1), Endpunkt: 3,8 (1,1); F=3,3, $\mathrm{p}=0,004$ );

- Funktionsniveau („Global Assessment of Functioning“ (GAF): Aufnahme: 37,0 (12,2), Endpunkt: 58,8 (14,4); $F=8,5$, $\mathrm{p}<0,001$ ),

- Lebensqualität (, quality of life enjoyment and satisfaction questionnaire“ (Q-LES-Q-18): Aufnahme: 2,2 (0,6), Endpunkt: $3,3(0,6) ; F=3,1, p=0,003$,) und
- Behandlungszufriedenheit („Client Satisfaction Questionnaire“ (CSQ-8): Aufnahme: 1,9 (0,4); Endpunkt: 3,2 (0,6); F=1,0, $\mathrm{p}<0,001)$.

Am 3-Jahres Endpunkt fanden sich größere Verbesserungen in der Psychopathologie $(F=15,2, p<0,001)$ und der Lebensqualität $(\mathrm{F}=5,8, \mathrm{p}=0,018$, ) in der BD- im Vergleich mit der SSD-Gruppe. Nach 36 Monaten wiesen 87 (75,7\%) Patienten eine volle medikamentöse Adhärenz auf, im Vergleich zu 29 Patienten $(25,2 \%)$ zu Beginn (McNemar's Test, $p<0,001$ ), es waren signifikant mehr Patienten beschäftigt als zu Beginn der Behandlung ( $n=38,33,0 \%$ vs. $n=21,18,3 \%$ zu Beginn; McNemar's Test, $\mathrm{p}<0,001)$.

\section{Zwangseinweisungen}

50 Patienten (43,5\%) wurden mind. einmal im Leben zwangseingewiesen, bei 34,8\% fand diese Zwangseinweisung in den letzten 2 Jahren vor Beginn der IV statt. Während der ersten 2 Jahre in der IV geschah dies bei nur noch $9(7,8 \%)$ und nach einem weite- 
ren Jahr bei insgesamt nur 11 (9,5\%) Patienten (McNemar's Test, $\mathrm{p}<0,001)$.

\section{Interventionen, Psychotherapie}

Die Inanspruchnahme von Psychotherapie stieg signifikant im Rahmen der Behandlung innerhalb der IV von 13\% (jemals erhaltene Psychotherapie, $n=15,13,0 \%)$ auf $73 \%(n=84$, McNemar's Test, $\mathrm{p}<0,001$ ).

\section{Ausblick \\ $\nabla$}

Die positiven Erfahrungen in der Behandlung von Patienten mit schweren psychischen Erkrankungen fließen aktuell in Hamburg in einem neuartigen BMBF-geförderten Projekt (Hamburger Netz Psychische Gesundheit [psychenet]) ein. Die IV mit ACT wurde auf Kinder- und Jugendliche im Alter von 12 bis 29 Jahren ausgeweitet und ein fächerübergreifendes Team implementiert. Dieses setzt oben genannte Behandlungsstrukturen bei Patienten mit einer psychotischen Ersterkrankung, unter Umgehung der künstlichen Grenze zwischen Kinder- und Jugend- und Erwachsenenpsychiatrie, um, womit Schnittstellenverluste und Behandlungsabbrüche beim Übergang ins Erwachsenenalter vermieden werden können. Ferner wurde ein fachübergreifendes Netzwerk geschaffen und kontinuierlich ausgebaut.

\section{Fazit für die Praxis}

Der Anteil an Patienten, die eine Psychotherapie erhielten, erhöhte sich signifikant, wie in der ACCESS-1-Studie ebenso gezeigt werden konnte, wobei dies einerseits ein Effekt des Bedarfs wie auch des Angebots von Psychotherapie in dieser Studie darstellen könnte.

Aus der klinischen Perspektive lassen sich 3 wesentliche Wirkelemente des Hamburger Modells identifizieren:

1) Die Behandlungskontinuität durch ein ACT Team über alle therapeutischen Settings,

2) ein multimodales und am individuellen Bedürfnis der Patienten adaptiertes Therapieangebot als Bestandteil der IV (inkl. Psychotherapie, Psychoedukation, Arbeitstherapie, Soziales-Kompetenz-Training, Suchtbehandlung, usw.) und

3) eine hohe therapeutische Spezialisierung des ACT Teams (inkl. störungsspezifischer Einzelpsychotherapie,

Familieninterventionen und Pharmakotherapie). Hinsichtlich der Stabilität der Behandlungsergebnisse und der Übertragbarkeit auf reale Alltagsbedingungen bietet die vorgestellte klinische Langzeituntersuchung erste vielversprechende Ergebnisse, wobei berücksichtigt werden muss, dass diese im Kontext eines urbanen Settings in einer Metropole erhoben wurden. Weitere Studienergebnisse werden in den folgenden Monaten publiziert werden. Aktuell liegen noch keine Ergebnisse zum neuen Ersterkrankten-IV-Projekt vor, welches jedoch wissenschaftlich evaluiert und begleitend beforscht wird.

Interessenkonflikt: Die Autoren geben an, dass kein Interessenkonflikt besteht.

\section{Literatur}

1 Riedel-Heller SG, Gühne U, Weinmann S et al. German Society for Psychiatry, Psychotherapy and Neurology. Psychosocial therapies in severe mental illness: DGPPN-S3-guideline: evidence, recommendations and challenges for mental health service research. Psychother Psychosom Med Psychol 2012; 62: 425-428

2 Lambert M, Bock T, Naber D et al. Mental Health of Children, Adolescents and Young Adults - Part 1: Prevalence, Illness Persistence, Adversities, Service use, Treatment Delay and Consequences. Fortschr Neurol Psychiatr 2013; 81: 614-627

3 Karow A, Bock T, Naber D et al. Mental health of children, adolescents and young adults - part 2: burden of illness, deficits of the german health care system and efficacy and effectiveness of early intervention services. Fortschr Neurol Psychiatr 2013; 81: 628-638

4 Schimmelmann $B$, Conus $P$, Cotton $S$ et al. Pre-treatment, baseline, and outcome differences between adolescent-onset and adult-onset psychosis in an epidemiological cohort of 636 first-episode patients. Schizophr Res 2007; 95: 1-9

5 Wang PS, Berglund P, Olfson $M$ et al. Failure and delay in initial treatment contact after first onset of mental disorders in the National Comorbidity Survey Replication. Arch Gen Psychiatry 2005; 62: 603-613

6 Robinson J, Cotton S, Conus P et al. Prevalence and predictors of suicide attempt in an incidence cohort of 661 young people with first-episode psychosis. Aust N Z J Psychiatry 2009; 43: 149-157

7 Marshall M, Lewis S, Lockwood A et al. Association between duration of untreated psychosis and outcome in cohorts of first-episode patients: a systematic review. Arch Gen Psychiatry 2005; 62: 975-983

8 Conus $P$, Lambert $M$, Cotton $S$ et al. Rate and predictors of service disengagement in an epidemiological first-episode psychosis cohort. Schizophr Res 2010; 118: 256-263

9 Lambert M, Bock T, Meigel-Schleiff $C$ et al. Integrierte Versorgung von Patienten mit psychotischen Erkrankungen nach dem Hamburger Modell: Rationalen, Behandlungsmodell und Ergebnisse der Vorstudie. Psychiatr Prax 2014; 41: 257-265

10 Karow A, Bock T, Meigel-Schleiff $C$ et al. Integrierte Versorgung von Patienten mit psychotischen Erkrankungen nach dem Hamburger Modell Teil 2: Ergebnisse des 2 und 4 Jahres Langzeitverlaufs. Psychiatr Prax 2014; 41: 266-273

11 Delespaul PH, de consensusgroep EPA. Consensus regarding the definition of persons with severe mental illness and the number of such persons in the Netherlands. Tijdschr Psychiatr 2013; 55: 427-438

12 Kreyenbuhl J, Nossel IR, Dixon LB. Disengagement from mental health treatment among individuals with schizophrenia and strategies for facilitating connections to care: a review of the literature. Schizophr Bull 2009; 35: 696-703

13 Lambert $M$, Conus $P$, Cotton $S$ et al. Prevalence, predictors, and consequences of long-term refusal of antipsychotic treatment in first-episode psychosis. J Clin Psychopharmacol 2010; 30: 565-572

14 Tiihonen J, Haukka J, Taylor M et al. A nationwide cohort study of oral and depot antipsychotics after first hospitalization for schizophrenia. Am J Psychiatry 2011; 168: 603-609

15 Velligan DI, Weiden PJ, Sajatovic $M$ et al. The expert consensus guideline series: adherence problems in patients with serious and persistent mental illness. J Clin Psychiatry 2009; 70 (Suppl 4): 1-46

16 Marshall $M$, Lockwood A. Assertive community treatment for people with severe mental disorders. Cochrane Database Syst Rev 2011, doi:10.1002/14651858.CD001089.pub2

17 Nordén T, Malm U, Norlander T. Resource Group Assertive Community Treatment (RACT) as a Tool of Empowerment for Clients with Severe Mental Illness: A Meta-Analysis. Clin Pract Epidemiol Ment Health 2012; 8: 144-151

18 Murphy $S$, Irving $C B$, Adams $C E$ et al. Crisis intervention for people with severe mental illnesses. Cochrane Database Syst Rev 2012, doi:10.1002/14651858.CD001087.pub4

19 Murphy $S$, Irving $C B$, Adams $C E$ et al. Crisis intervention for people with severe mental illnesses. Schizophr Bull 2012; 38: 676-677

20 Dieterich $M$, Irving $C B$, Park $B$ et al. Intensive case management for severe mental illness. Cochrane Database Syst Rev 2010, doi:10.1002/14651858.CD007906.pub2

21 Malone D, Marriott S, Newton-Howes $G$ et al. Community mental health teams for people with severe mental illnesses and disordered personality. Schizophr Bull 2009; 35: 13-14

22 Schöttle D, Karow A, Bock $T$ et al. Integrated Care in patients with schizophrenia: results of trials published between 2011 and 2013 focusing on effectiveness and efficiency. Curr Opin Psychiatry 2013; 26: $384-408$ 
23 Schöttle D, Bock T, Meigel-Schleiff C et al. Translating research into clinical practice: effectiveness of Integrated Care (IC) including therapeutic Assertive Community Treatment (ACT) in severe and persistent schizophrenia-spectrum and bipolar I disorders - a 24 months follow-up study (ACCESS II study). J Clin Psychiatry [in press]

24 Teague GB, Bond GR, Drake RE. Program fidelity in assertive community treatment: development and use of a measure. Am J Orthopsychiatry 1998; 68: 216-232

25 Guy W. Clinical global impression. In: Guy W. (ed.). ECDEU Assessment Manual for Psychopharmacology, revised. Rockville, MI: National Insitute of Mental Health, 1976; 217-222

26 Spearing $M K$, Post RM, Leverich GS et al. Modification of the Clinical Global Impression (CGI) scale for use in bipolar illness (BP): The CGIBP. Psychiatry Research 1997; 73: 159-171

27 American Psychiatric Association. DI. American Psychiatric Association and Task Force on DSM IV. Diagnostic and Statistical Manual of Mental Disorders, DSM IV. 4 ed. Washington, DC: APA, 1994
28 Overall J, Gorham $D$. The brief psychiatric rating scale. Psychological Reports 1962; 10: 799-812

29 Tohen M, Hennen J, Zarate CM et al. Two-year syndromal and functional recovery in 219 cases of first-episode major affective disorder with psychotic features. Am J Psychiatry 2000; 157: 220-228

30 Ritsner M, Kurs R, Gibel A et al. Validity of an abbreviated quality of life enjoyment and satisfaction questionnaire (Q-LES-Q-18) for schizophrenia, schizoaffective, and mood disorder patients. Qual Life Res 2005; 14: 1693-1703

31 Nguyen TD, Attkisson CC, Stegner BL. Assessment of patient satisfaction: Development and refinement of a service evaluation questionnaire. Eval Program Plann 1983; 6: 299-314 